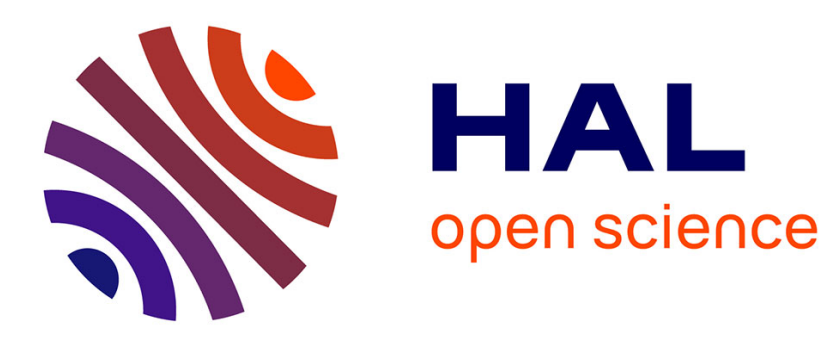

\title{
Identification of two allotwins of mica polytypes in reciprocal space through the minimal rhombus unit
}

Massimo Nespolo, Giovanni Ferraris, Hiroshi . Takeda

\section{To cite this version:}

Massimo Nespolo, Giovanni Ferraris, Hiroshi . Takeda. Identification of two allotwins of mica polytypes in reciprocal space through the minimal rhombus unit. Acta Crystallographica Section B: Structural Science, Crystal Engineering and Materials [2014-..], 2000, B56, pp.639-647. 10.1107/S0108768100002044 . hal-00130624

\section{HAL Id: hal-00130624 https://hal.science/hal-00130624}

Submitted on 13 Feb 2007

HAL is a multi-disciplinary open access archive for the deposit and dissemination of scientific research documents, whether they are published or not. The documents may come from teaching and research institutions in France or abroad, or from public or private research centers.
L'archive ouverte pluridisciplinaire HAL, est destinée au dépôt et à la diffusion de documents scientifiques de niveau recherche, publiés ou non, émanant des établissements d'enseignement et de recherche français ou étrangers, des laboratoires publics ou privés. 


\title{
Identification of two allotwins of mica polytypes in reciprocal space through the minimal rhombus unit
}

\author{
Massimo Nespolo, Giovanni Ferraris and Hiroshi Takeda
}


Acta Crystallographica Section B

\section{Structural}

Science

ISSN 0108-7681

\section{Massimo Nespolo, ${ }^{\mathrm{a} *}$ Giovanni Ferraris $^{b}$ and Hiroshi Takeda ${ }^{c}+$}

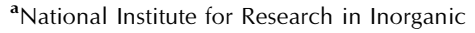 \\ Materials, Research Centre for Creating New \\ Materials,1-1 Namiki, Tsukuba, 305-0044 \\ Ibaraki, Japan, 'buniversity of Torino, Depart- \\ ment of Mineralogical and Petrological \\ Sciences, via Valperga Caluso 35, 10125 \\ Torino, Italy, and ${ }^{\mathbf{c}}$ University of the Ryukyus, \\ Faculty of Science, Department of Physics and \\ Earth Sciences, 1-Senbaru, Nishihara, Naka- \\ gami-Gun, 903-0213 Okinawa, Japan
}

+ Present address: Chiba Institute of Technology, Research Institute, 2-17-1 Tsudanuma, Narashino, Chiba 275-0016, Japan.

Correspondence e-mail: nespolo@nirim.go.jp

(C) 2000 International Union of Crystallography Printed in Great Britain - all rights reserved

\section{Identification of two allotwins of mica polytypes in reciprocal space through the minimal rhombus unit}

The X-ray investigation (precession method) of the Ruiz Peak oxybiotite, which is well known for the occurrence of a large number of polytypes and twins, revealed two complex diffraction patterns, which cannot be identified as long-period polytypes. These patterns are analysed in terms of the minimal rhombus, a geometrical asymmetric unit in reciprocal space which permits the decomposition of the composite reciprocal lattice of a twin or allotwin into the reciprocal lattices of the individuals. Both the recorded patterns correspond to a $1 M-$ $2 M_{1}$ allotwin: the relative rotation between the individuals is $120^{\circ}$ in one case and $60^{\circ}$ in the other. The geometrical criteria for evaluating the presence of twinning or allotwinning are analysed through these two natural examples.

\section{Introduction}

Mica polytypes represent one of the most complex series of inorganic structures known to date (Smith \& Yoder, 1956; Ross et al., 1966; Rieder, 1970; Nespolo \& Takeda, 1999; Kogure \& Nespolo, 1999). They also form different types of oriented crystal associations: twins and plesiotwins (same chemical composition and same polytype; Nespolo, Ferraris, Takeda \& Takéuchi, 1999), allotwins (same chemical composition but different polytypes; Nespolo, Kogure \& Ferraris, 1999), epitaxy (oriented overgrowth with different chemical compositions; Royer, 1928, 1954), and syntaxy (oriented intergrowth with different chemical compositions; Ungemach, 1935).

Polytypism and twinning are two phenomena resulting from the presence of higher-symmetrical units or subunits within the normal unit cell (Ito, 1950). In the case of micas, since the relative rotations between individuals ${ }^{\mathbf{1}}$ in a twin are practically identical to the relative rotations between layers in a polytype, namely $n \times 60^{\circ}(0 \leq n \leq 5)$, the diffraction pattern of a mica twin can be mistaken for that of a single crystal corresponding to a polytype with longer periods. Takano \& Takano (1958) introduced the definitions of 'real polytypism' and 'apparent polytypism', to distinguish true polytypes from twins that can be mistaken for polytypes. The general criteria for distinguishing between real polytypism and apparent polytypism in micas are given in Nespolo (1999) and are applicable when the geometry of the diffraction pattern is not exactly identical in the two cases, namely (with rare exceptions) in twinning by reticular pseudo-merohedry, which is the most common twinning in micas. Application of these criteria showed (Nespolo \& Takeda, 1999) that the 24-layer polytype reported

\footnotetext{
${ }^{\mathbf{1}}$ The term 'individual' is used here to indicate one crystal of a twin or allotwin, and the term 'single crystal' to represent an untwinned crystal. Other authors (e.g. Hahn et al., 1999) use 'component' instead of 'individual'.
}

Received 10 December 1999 Accepted 4 February 2000 
by Hendricks \& Jefferson (1939) was not a twin of an eightlayer polytype, as previously stated by Smith \& Yoder (1956).

Nespolo et al. (2000) developed a theory to geometrically analyse the diffraction pattern of composite crystals and decompose the twin-/allotwin-weighted reciprocal lattice (w.r.l.) into the w.r.l. of the individuals, obtaining the relative orientations of the individuals. These orientations depend upon the twin/allotwin laws, which however have a non-1:1 correspondence: more than one law may correspond to the same orientation as the individuals. A thorough identification of the twin/allotwin laws requires a morphological analysis and will be studied elsewhere.

In the course of a single-crystal X-ray diffraction study of oxybiotite samples from the Ruiz Peak rhyodacite ash flow, Jemez Mountains (New Mexico), two crystals giving complex diffraction patterns have been found. The analysis of their diffraction pattern is presented, preceded by a brief summary of the relevant theoretical tools.

\section{Theoretical background}

Mica polytypes are built by stacking along the $c$ direction a layer ( $M$ layer) about $10 \AA$ thick. The thickness of the layer is also the period along $c$ for the $1 M$ polytype $\left(c_{1}\right)$. The corresponding reciprocal space period along $c^{*}$ is labelled $c^{*}{ }_{1}$ (about $0.1 \AA^{-1}$ ). In the diffraction pattern of an $N$-layer polytype, $N$ reflections appear in the $c^{*}{ }_{1}$ repeat along the nonfamily rows (see below) parallel to $c^{*}$.

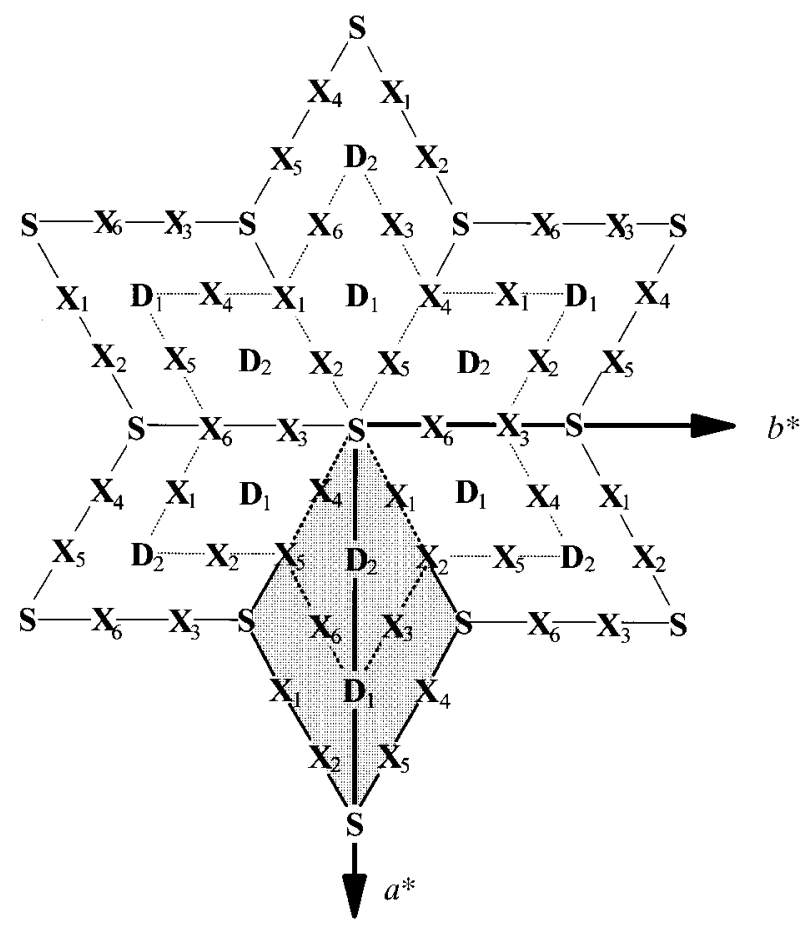

Figure 1

Minimal rhombus (dotted lines; in the foreground) and tessellation rhombus (solid lines) in the six orientations defining the star polygon. The nine translationally independent rows are distinguished by type and sequence numbers $\left(\mathrm{S} ; \mathrm{D}_{1}-\mathrm{D}_{2} ; \mathrm{X}_{1}-\mathrm{X}_{6}\right)$ (modified after Nespolo et al., 2000).
All mica polytypes have an (ideally) orthohexagonal axial setting $C_{1}$ (Nespolo et al., 1998) based on two (pseudo)orthohexagonal axes $(a, b)$ in the (001) plane, for which the orthohexagonal relation

$$
b=a 3^{1 / 2} ; \quad \gamma=90^{\circ}
$$

is approximately obeyed. The $a$ and $b$ axes define the two shortest mutually orthogonal translations in the plane of the layer. On the basis of the projection $\mathbf{c}_{n}$ of the $c$ axis onto the (001) plane, mica polytypes are divided into three types:

(i) orthogonal polytypes: $\mathbf{c}_{n}=(0,0)$;

(ii) Class a polytypes: $\mathbf{c}_{n}=(-1 / 3,0)$;

(iii) Class b polytypes: $\mathbf{c}_{n}=(0,-1 / 3)$.

The number of layers $N$ in a mica polytype is suitably expressed by the following relationship between integers

$$
N=3^{n}(3 K+L) \text {, }
$$

where $n \geq 0$ defines the Series, $L=1,2$ the Subclass and $K \geq 0$ appears in the transformation matrices between axial settings.

The diffraction pattern of a twinned mica polytype belonging to Series $n$ can show the same number of reflections as that of an untwinned mica polytype belonging to Series $n+1$. In fact, the cell of the twin lattice coincides with the cell of the (pseudo-)orthohexagonal setting defined by (1). For nonorthogonal polytypes the period in this setting is $3 N c_{0}\left(c_{0}=\right.$ $\left.1 / c^{*}{ }_{1}<c_{1}\right)$ : depending on the number of individuals and their relative rotations, the diffraction pattern shows $3 N$ equally spaced reflections in the $c^{*}{ }_{1}$ repeat, exactly as in the case of an untwinned $3 \mathrm{~N}$-layer polytype. However, the location of these reflections, in particular their normal projection onto the $c^{*}$ axis, in most cases permits to discriminate between real and apparent polytypism. Three types of reciprocal lattice rows are involved.

(i) $\mathbf{S}$ rows, corresponding to $h=0(\bmod 3)$ and $k=0(\bmod 3)$;

(ii) D rows, corresponding to $h \neq 0(\bmod 3)$ and $k=$ $0(\bmod 3)$;

(iii) $\mathbf{X}$ rows, corresponding to $k \neq 0(\bmod 3)$,

where S, D and X represent 'Single', 'Double' and 'Sextuple', respectively. These three types of rows are translationally independent and are related by $n \times 60^{\circ}$ rotations about $c^{*}$ (Nespolo et al., 1997; Nespolo et al., 2000). All the central reciprocal lattice planes containing $c^{*}$ can be divided into two types: SD planes (containing only S and D rows) and SX planes (containing only $\mathrm{S}$ and $\mathrm{X}$ rows).

All polytypes of a substance built on the same structural principle are $\mathrm{OD}^{2}$ polytypes and have a common set of reflections, called family reflections (Dornberger-Schiff \& Fichtner, 1972), which are always sharp. Along family rows, non-space-group absences appear, which derive from the existence of local symmetry operations (space groupoid operations; Sadanaga \& Ohsumi, 1979; Fichtner, 1980) relating pairs of layers. The OD character of the polytype determines the number and disposition of reflections along $\mathrm{S}$, D and X rows (Ďurovič et al., 1984; Nespolo, 1999).

\footnotetext{
${ }^{2}$ OD represents 'order-disorder'. However, OD phenomena refer to the geometrical equivalence of layer pairs and are of completely different nature from the structural order-disorder phenomena.
} 


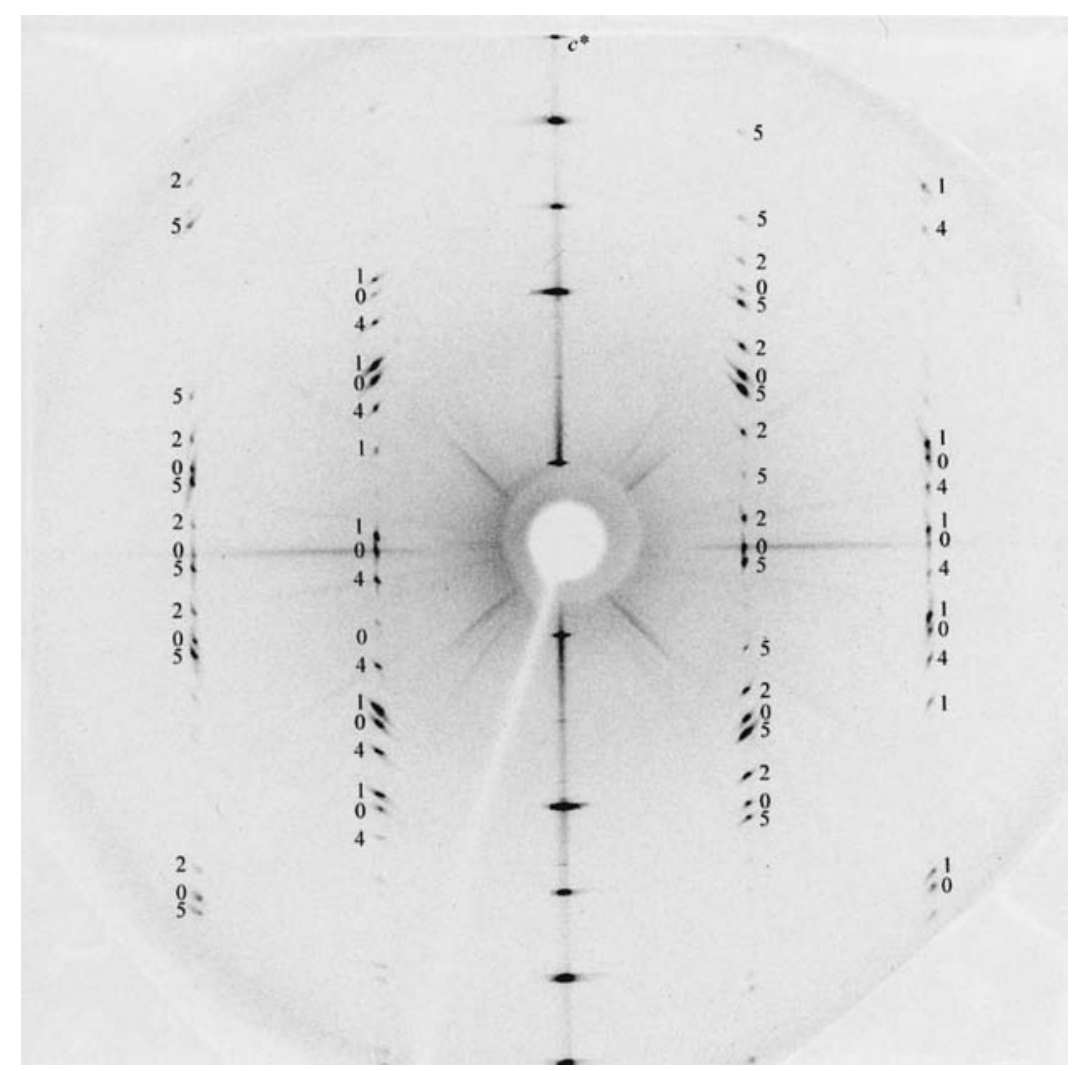

\section{Figure 2}

Precession diffraction pattern from the first SX plane (SX1) of the sample No. 1. The $l$ index of the reflections is expressed $(\bmod 6)$.

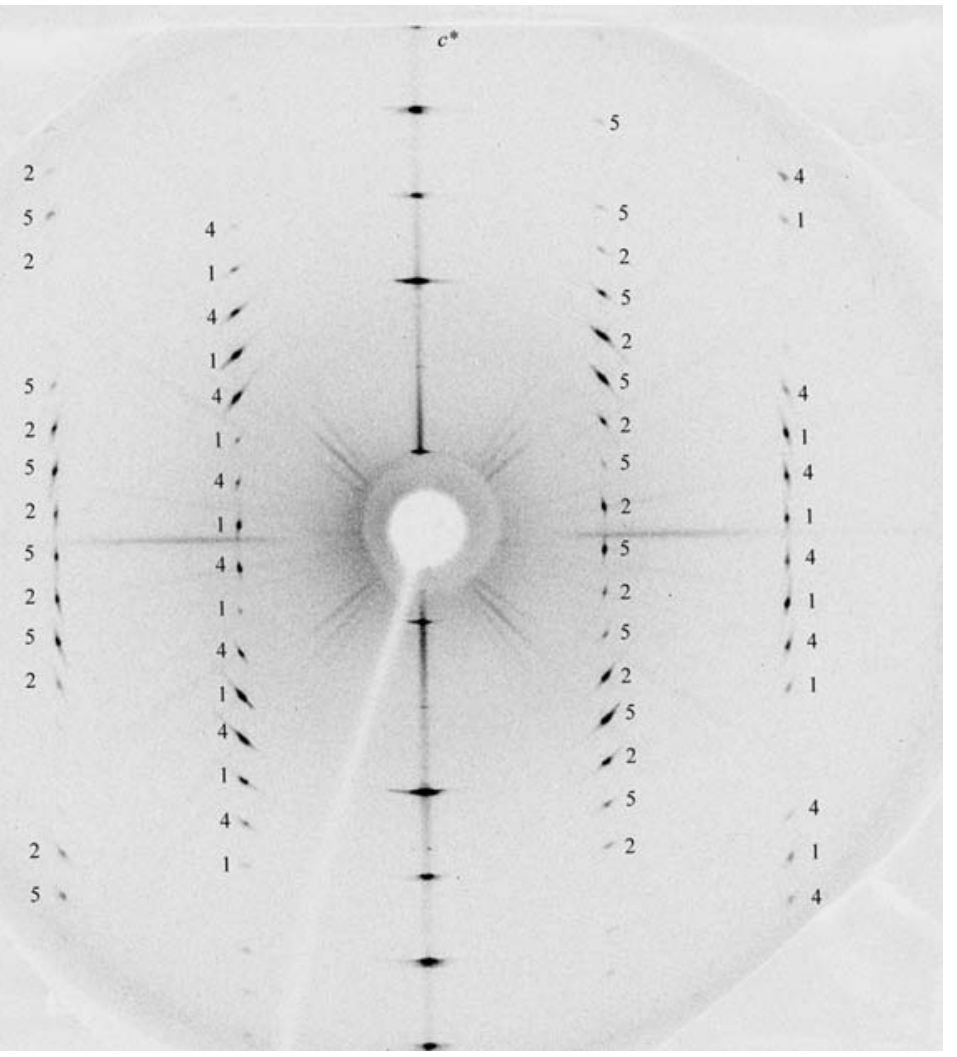

Figure 3

Precession diffraction pattern from the second SX plane (SX2) of the sample No. $1\left(60^{\circ}\right.$ from SX1). The $l$ index is as given in Fig. 2.
S rows are family rows common to all polytypes. On them, a single reflection appears in the $c^{*}{ }_{1}$ repeat, with the presence criterion $l=0(\bmod N)$. The nonspace-group absences along $\mathrm{D}$ rows depend upon the type of local symmetry operations relating layer pairs. Accordingly, polytypes of micas are classified into three types: subfamily A polytypes $(2 n \times$ $60^{\circ}$ only), subfamily B polytypes [ $(2 n+1)$ $\times 60^{\circ}$ only] and mixed-rotation polytypes [both $2 n \times 60^{\circ}$ and $(2 n+1) \times 60^{\circ}$ ]. Along $\mathrm{D}$ rows, the number of reflections is 1 for subfamily A [presence criterion $l=$ $( \pm h / 3)(\bmod 3)]$, two for subfamily B [equally spaced, at $l=0(\bmod N)$ and $l=$ $N / 2(\bmod N): N$ is necessarily even in subfamily B polytypes] and $N$ for mixedrotation polytypes. Finally, $\mathrm{X}$ rows are non-family rows for all polytypes and on them $N$ reflections appear in the $c^{*}{ }_{1}$ repeat.

The reciprocal lattice of a mica twin or allotwin is characterized by a tessellation rhombus (Fig. 1, solid lines) on whose edges and diagonals are nine translationally independent rows: one $\mathrm{S}$, two $\mathrm{D}$ and six X. The whole reciprocal space can be tessellated by $(3 p, 3 q)$ translations $(p, q$ integers of the same parity) along $\mathbf{a}^{*}$ and b*. Inside the tessellation rhombus a smaller unit, termed the minimal rhombus, defined by the same row, can be drawn (Fig. 1, dotted lines). There are six possible orientations of the tessellation rhombus and of the minimal rhombus, related by $n \times 60^{\circ}$ rotations $(0 \leq n \leq 5)$, which, however, represent equivalent descriptions of the same reciprocal lattice. The rows that can be reached by rotating the original rhombus are within a starpolygon located by the six rhombi with the common origin.

The nine translationally independent rows can be experimentally inspected by recording four central planes: one SD plane and three SX planes each $60^{\circ}$ apart. Then the polytypes involved in a twin or allotwin and the relative rotations between them are obtained by comparing the experimental minimal rhombus with those computed (Nespolo et al., 2000) by combining the minimal rhombi of the single crystals in all the independent orientations. The relative orientations are then expressed through the so-called $Z_{T}$ symbols: $Z_{T}=Z_{1} Z_{2} \ldots Z_{N}\left(1 \leq Z_{i} \leq 6\right)$ for a 


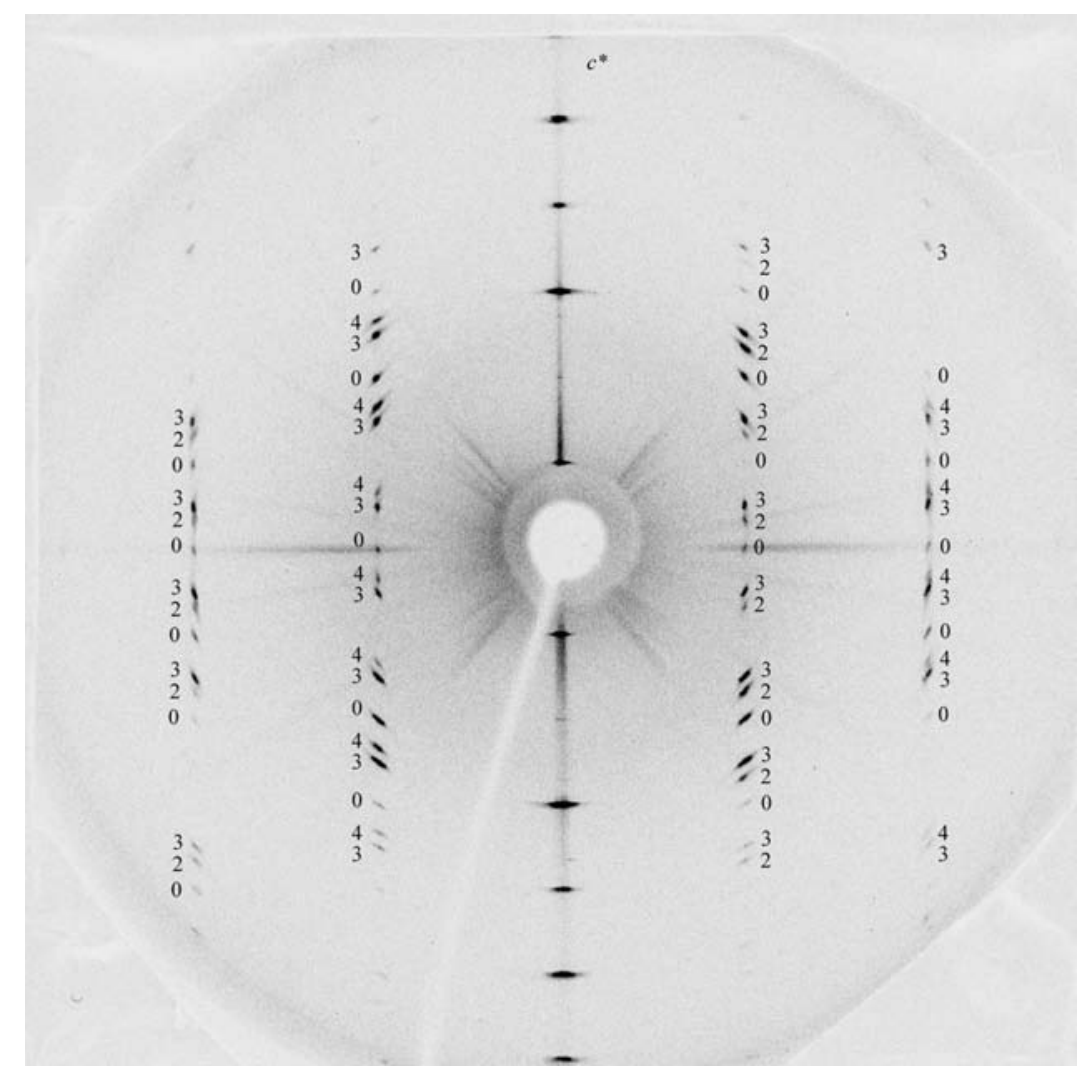

Figure 4

Precession diffraction pattern from the third SX plane (SX3) of the sample No. $1\left(120^{\circ}\right.$ from SX1). The $l$ index is as given in Fig. 2.

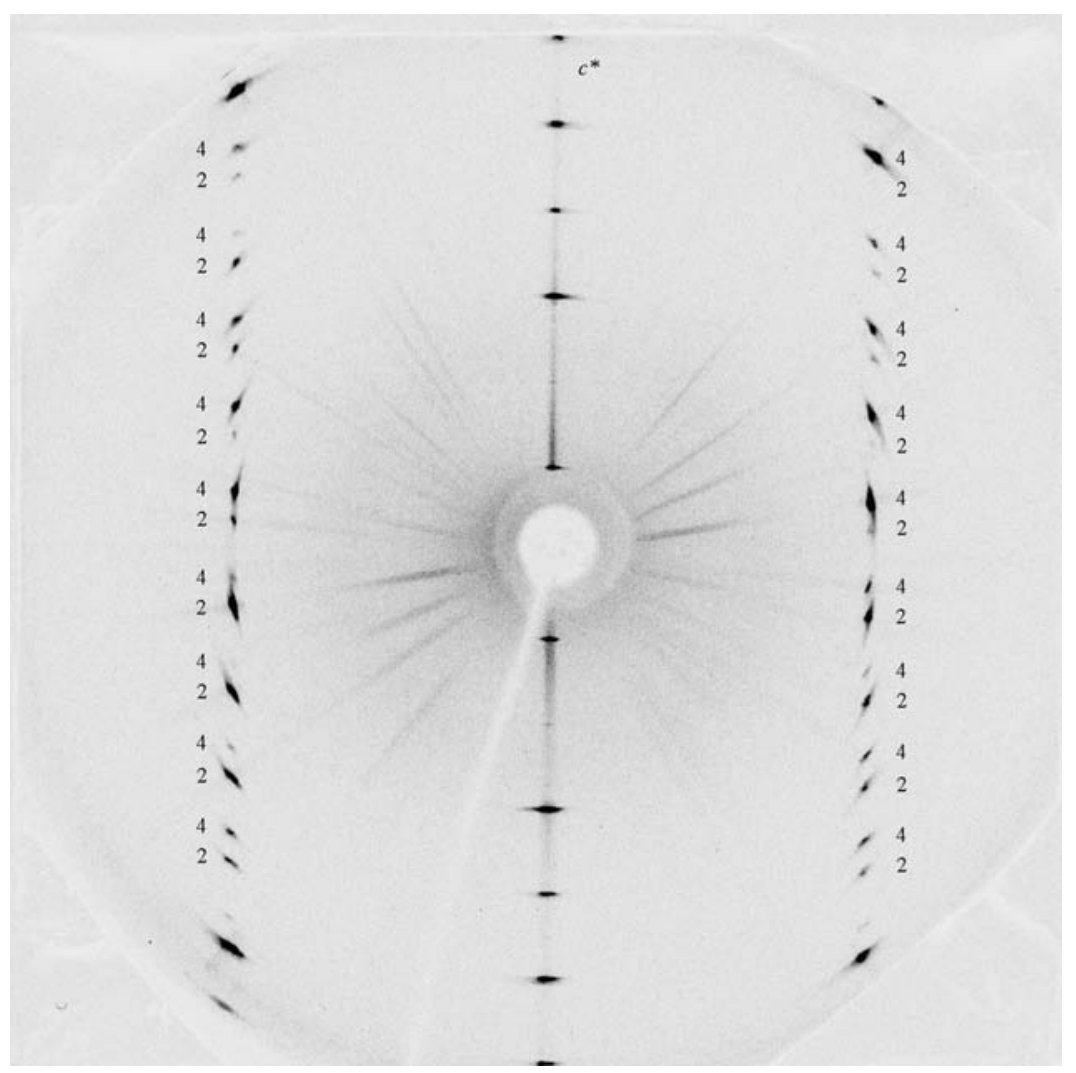

Figure 5

Precession diffraction pattern from an SD plane of the sample No. 1 ( $30^{\circ}$ from SX3). The $l$ index is as given in Fig. 2. twin composed of $N$ individuals $(N=2,6)$ and $Z_{T}=Z_{1} Z_{2} \ldots Z_{N} Z_{1} Z_{2} \ldots Z_{N}$ for a binary allotwin with $N$ and $N^{\prime}$ individuals (for details refer to Nespolo et al., 2000).

\section{Materials and methods}

The samples investigated are oxybiotite crystals from Ruiz Peak, New Mexico (Smith et al., 1961; Bailey \& Smith, 1978). Ohta et al. (1982) reported the composition as $\left(\mathrm{K}_{0.773} \mathrm{Na}_{0.162} \mathrm{Ba}_{0.022}\right)\left(\mathrm{Mg}_{1.666} \mathrm{Fe}_{0.013}{ }^{2+}\right.$. $\left.\mathrm{Fe}_{0.855}{ }^{3+} \mathrm{Mn}_{0.010} \mathrm{Ti}_{0.342} \mathrm{Al}_{0.163}\right)\left(\mathrm{Al}_{1.159} \mathrm{Si}_{2.841}\right)$ $\mathrm{O}_{11.620} \mathrm{~F}_{0.168}(\mathrm{OH})_{0.212}$. According to Rieder et al. (1998) it should be renamed 'ferric phlogopile': here the original name 'oxybiotite' is retained for the sake of comparison with previous studies on the same sample.

The sample, formed by vapour growth in the cavities of lava at high temperature, has $\mathrm{Fe}^{2+}$ almost completely oxidized to $\mathrm{Fe}^{3+}$ and a considerable amount of Ti: correspondingly, $\mathrm{OH}^{-}$is largely substituted by $\mathrm{O}^{2-}$ and consequently $\mathrm{OH}+\mathrm{F}=0.38$ rather than 2.0. The presence of $\mathrm{Ti}$ is responsible for the brown-reddish colour of the crystals (Robbins \& Strens, 1972; for further details on the sample, refer to Ross \& Wones, 1965; Ross et al., 1966; Takeda \& Ross, 1975; Ohta et al., 1982). In this mica several polytypes and twins were reported (Ross et al., 1966; Takeda \& Ross, 1995; Nespolo \& Takeda, 1999; Kogure \& Nespolo, 1999).

The crystals, selected by optical microscopy, have been mounted on a glass fibre, with $c^{*}$ parallel to the rotation axis of the goniometer head of a Buerger precession camera (Charles Supper Co., model 3000, series 7426). Ni-filtered $\mathrm{Cu} K \alpha$ radiation was used, with a $35 \mathrm{kV}$ operating voltage and $15 \mathrm{~mA}$ current. Diffraction was recorded on an imaging plate (Fujifilm BAS-RS 127) and transformed into digital images by a Rigaku R-AXIS DS2 laser reader.

The reflections seen in the precession photographs have oval shape and are rather broad and streaked. Micas almost invariably give this type of reflection, because of their platy morphology, which often is deformed and kinked (Hörz, 1970; Bell et al., 1986). The poor quality of the diffraction is a severe obstacle to the structure refinement of micas. However, the purpose of the present research is to identify the polytypes and their relative rotations: a pure geometrical analysis of the 
diffraction pattern, in terms of presence/absence criteria, is sufficient and also reduction for Lorentz-polarization and absorption is not required. Besides, because of the rarity of the phenomenon observed, the probability of finding better crystals is low.

\section{Results}

Two of the samples investigated gave a complex diffraction pattern, which cannot correspond to a single crystal of a longperiod polytype. Figs. 2-5 and 7-10 show, for each sample, the diffraction patterns from the four central planes (one SD and three SX) necessary to obtain the minimal rhombus. In the investigation of micas by the precession method six central planes can be recorded at rotation intervals of $30^{\circ}$ (Takeda \& Ross, 1995): three of type SD $[(h 0 l),(h .3 h . l),(h .3 \bar{h} . l)]$ and three of type SX $[(0 k l),(h h l),(h \bar{h} l)]$. In twins, the diffraction pattern corresponds to the overlap of the reciprocal lattices of the individuals: hereinafter the SD plane recorded is assumed to coincide with the $(h 0 l)$ plane in the twin setting (space-fixed reference). Correspondingly, the experimental minimal rhombus is assumed to have its long diagonal coincident with $a^{*}$ of the twin setting.

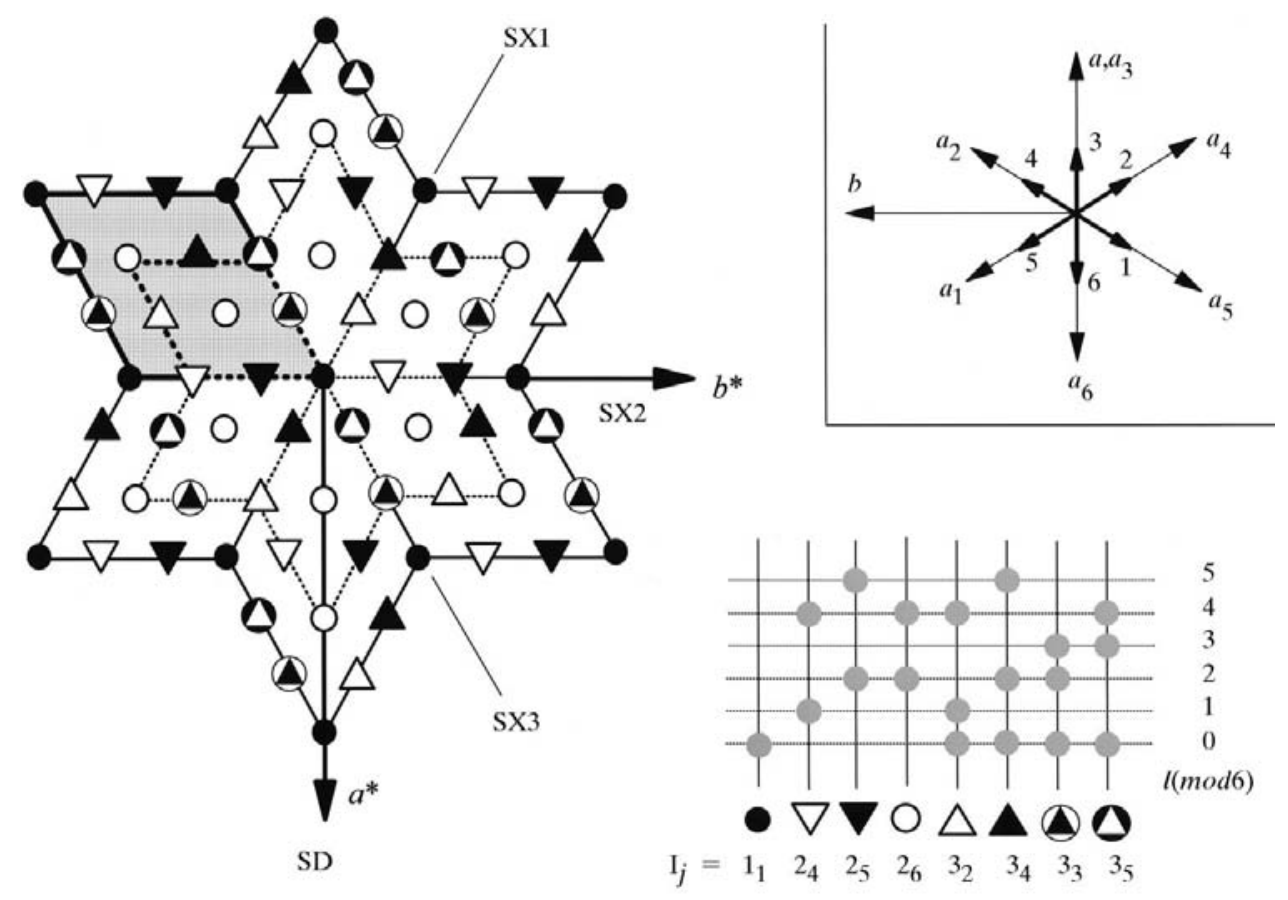

Figure 6

Construction of the star polygon corresponding to the diffraction patterns in Figs. 2-5. The SD plane in Fig. 2 is taken to be coincident with the $\left(a^{*}, c^{*}\right)$ plane and the three SX planes are reported to be counter clockwise, according to the rotations shown in Figs. 2-4. The star polygon is then obtained by $(3 p, 3 q)$ translations of the nine translationally independent rows in these four planes. The minimal rhombus and the tessellation rhombus are indicated in their six possible orientations. The shaded minimal rhombus corresponds to the $Z_{T}={ }_{5}^{3}$ minimal rhombus tabulated in Nespolo et al. (2000). Inset on the top-right: axes $(a, b)$ of the space-fixed reference and of the individual-fixed references in the six possible orientations $\left(a_{1}-\right.$ $\left.a_{6}\right)$, and corresponding $Z_{T}$ symbols $\left(b_{1}-b_{6}\right.$ axes are not shown). Inset in the bottom-right: $l(\bmod 6)$ indices of the reflections which are present on the composite rows of the lattice, and symbol of the rows. $I_{j}$ is the symbol identifying the composite row, where $I$ gives the number of reflections in the $c^{*}{ }_{1}$ repeat and $j$ is a sequence number $\left(I_{j}\right.$ are defined and tabulated in Nespolo et al., 2000)

\subsection{Sample 1: $1 M-2 M_{1}$ allotwin $Z_{T}={ }^{3}$}

Figs. 2-4 are the diffraction patterns from three SX planes each $60^{\circ}$ apart. The shortest separation between successive reflections along $c^{*}$ of $\mathrm{X}$ rows is $c^{*} 1 / 6$ : the apparent period is six layers and thus the $l$ index of all the reflections are expressed (mod 6). Fig. 5 shows the diffraction pattern from an SD plane: two strong reflections appear along D rows, corresponding to $l=2(\bmod 6)$ and $l=4(\bmod 6)$, whereas no reflections appear at $l=0(\bmod 6)$. This type of diffraction equivocally originates from a twin or allotwin of subfamily A polytypes, with a relative rotation between the individuals that produces a separation of the family reflections along D s. It follows that the apparent six-layer period along $\mathrm{X}$ is actually due to the twinning of a two-layer polytype. only two-layer subfamily A polytype is $2 M_{1}$ and the (win of $2 M_{1}$ or an allotwin involving, these cases and to identify the relative rotations, the minimal mbus analysis (Nespolo et al., 2000) is necessary.

Fig. 6 shows the experimental star polygon, built by the six possible orientations of the tessellation rhombus and the minimal rhombus, drawn by reporting the $l(\bmod 6)$ indices of the reflections occurring in the four diffraction patterns, and applying the $(3 p, 3 q)$ translations between translationally equivalent reciprocal lattice rows. None of the six orientations of the minimal rhombus matches any of the nine independent minimal rhombi which are possible for the $2 M_{1}$ twins and are reported in Nespolo et al. (2000). The sample is thus an allotwin. It cannot involve a $3 T$ crystal, otherwise three reflections corresponding to $l=0(\bmod 6), l=2(\bmod 6)$ and $l=$ $4(\bmod 6)$ would appear along all $\mathrm{X}$ rows. The sample is thus a $1 M-$ $2 M_{1}$ allotwin. The shaded minimal rhombus matches the computed minimal rhombus of the $1 M-2 M_{1}$ allotwin with a relative rotation of $120^{\circ}$ between the two individuals and corresponds to $Z_{T}={ }^{3}$ in Nespolo et al. (2000).

A binary allotwin is characterized by two allotwin indices: for a $1 M-2 M_{1}$ allotwin, the allotwin index of $1 M\left(2 M_{1}\right)$ is defined as the ratio of the total number of lattice nodes of $1 M\left(2 M_{1}\right)$ to the number of lattice nodes common to $1 M$ and $2 M_{1}$ (Nespolo, Kogure $\&$ Ferraris, 1999). The cell of the allotwin lattice is the orthogonal 


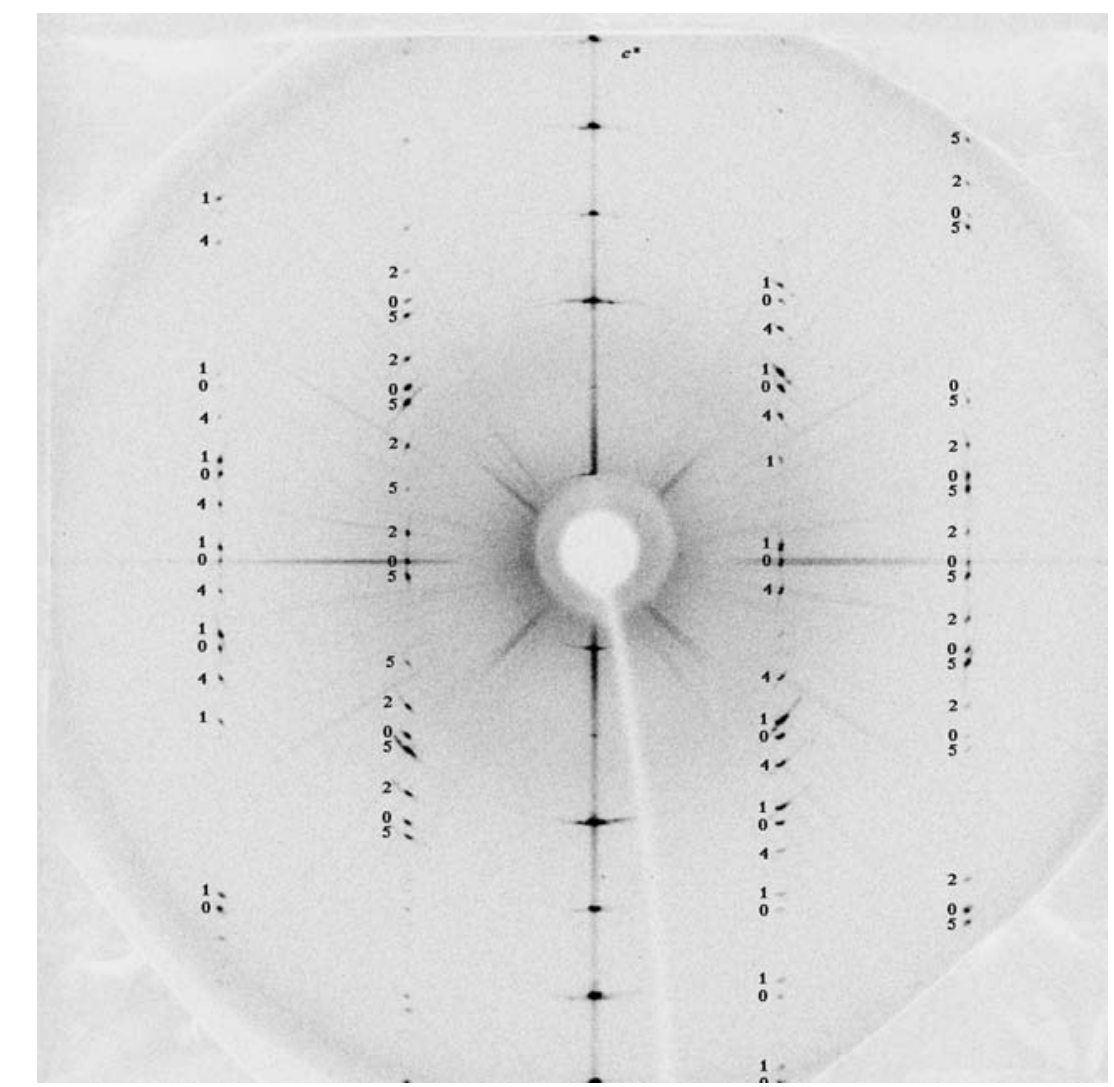

Figure 7

Precession diffraction pattern from the first SX plane (SX1) of the sample No. 2. The $l$ index is as given in Fig. 2.

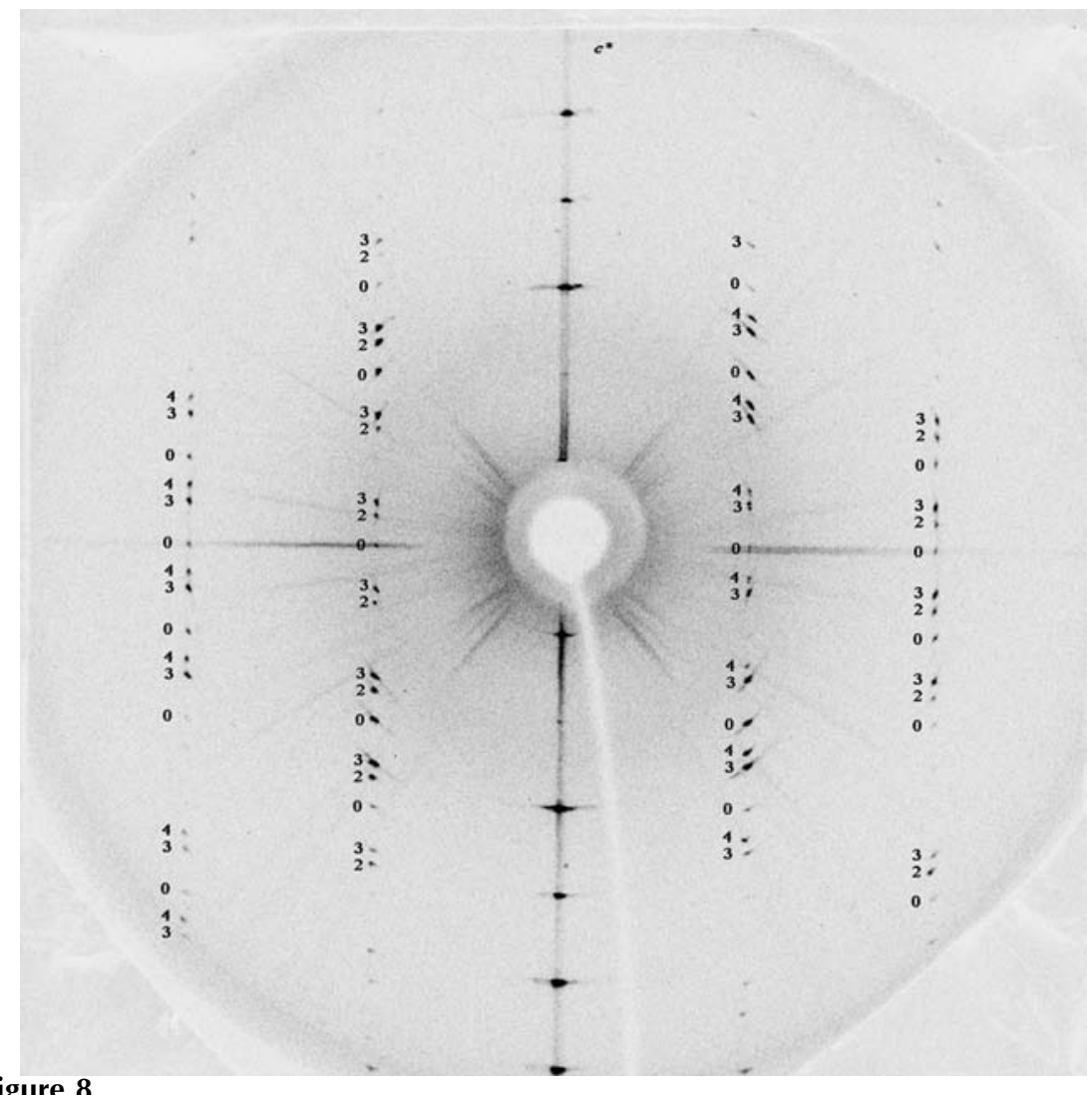

Precession diffraction pattern from the second SX plane (SX2) of the sample No. $2\left(60^{\circ}\right.$ from SX1). The $l$ index is as given in Fig. 2. cell common to both polytypes: its period along $c$ is $6 c_{1}$ and thus contains six lattice planes of the $1 M$ polytype and three lattice planes of the $2 M_{1}$ polytype. The $z$ coordinates of the (001) lattice planes for the two polytypes are $z=n(\bmod 6) / 6$ for $1 M$ and $z=2 n(\bmod 6) / 6$ for $2 M_{1}(n$ integer $)$. Of these, one $[z=0(\bmod 6)]$ has all the nodes from both polytypes overlapped by the allotwin operation, whereas in all the other lattice planes the nodes from the two polytypes are distinct. Consequently, the allotwin index of $1 M$ is 6 and that of $2 M_{1}$ is 3.

The case illustrated here can correspond both to a rotation allotwin, with allotwin law [310] and composition plane (001), and to a reflection allotwin, with allotwin and composition plane (110). Besides, the first individual (higher digit in the $Z_{T}$ symbol) could correspond to $2 M_{1}$ rather than $1 M$, a situation physically indistinguishable from the allotwin $Z_{T}=$ ${ }_{1}^{3}$, which gives an equivalent diffraction pattern, but corresponds to the allotwin laws [310] (rotation allotwin) or (11̄0) (reflection allotwin).

\subsection{Sample 2: $1 M-2 M_{1}$ allotwin $Z_{T}={ }_{4}^{3}$}

Figs. 7-9 are the diffraction patterns from three SX planes of sample 2. As in the previous case, the shortest separation between successive reflections along $X$ rows is $c^{*}{ }_{1} / 6$. Fig. 10 shows the diffraction pattern from an SD plane of the same sample which, with one reflection for the $c^{*}{ }_{1}$ repeat, has the typical appearance of a subfamily A polytype. The presence of twinning is not at all evident from this plane.

In principle, the investigated sample could be either a six-layer polytype, or a twin or allotwin involving the $2 M_{1}$ polytype: therefore, the possibility of apparent polytypism has to be taken into account. However, two of the SX planes (Figs. 7 and 8) are of orthogonal type (i.e. reflections are present at $l=0$ along each row parallel to $\left.c^{*}\right)$. This geometry of the reciprocal lattice is impossible for a six-layer subfamily A polytype, which would belong to Class $b$ and should therefore have all the SX planes non-orthogonal (Nespolo, 1999). It also follows that in this case the sample is a twin or allotwin of the $2 M_{1}$ polytype. The corresponding star polygon 


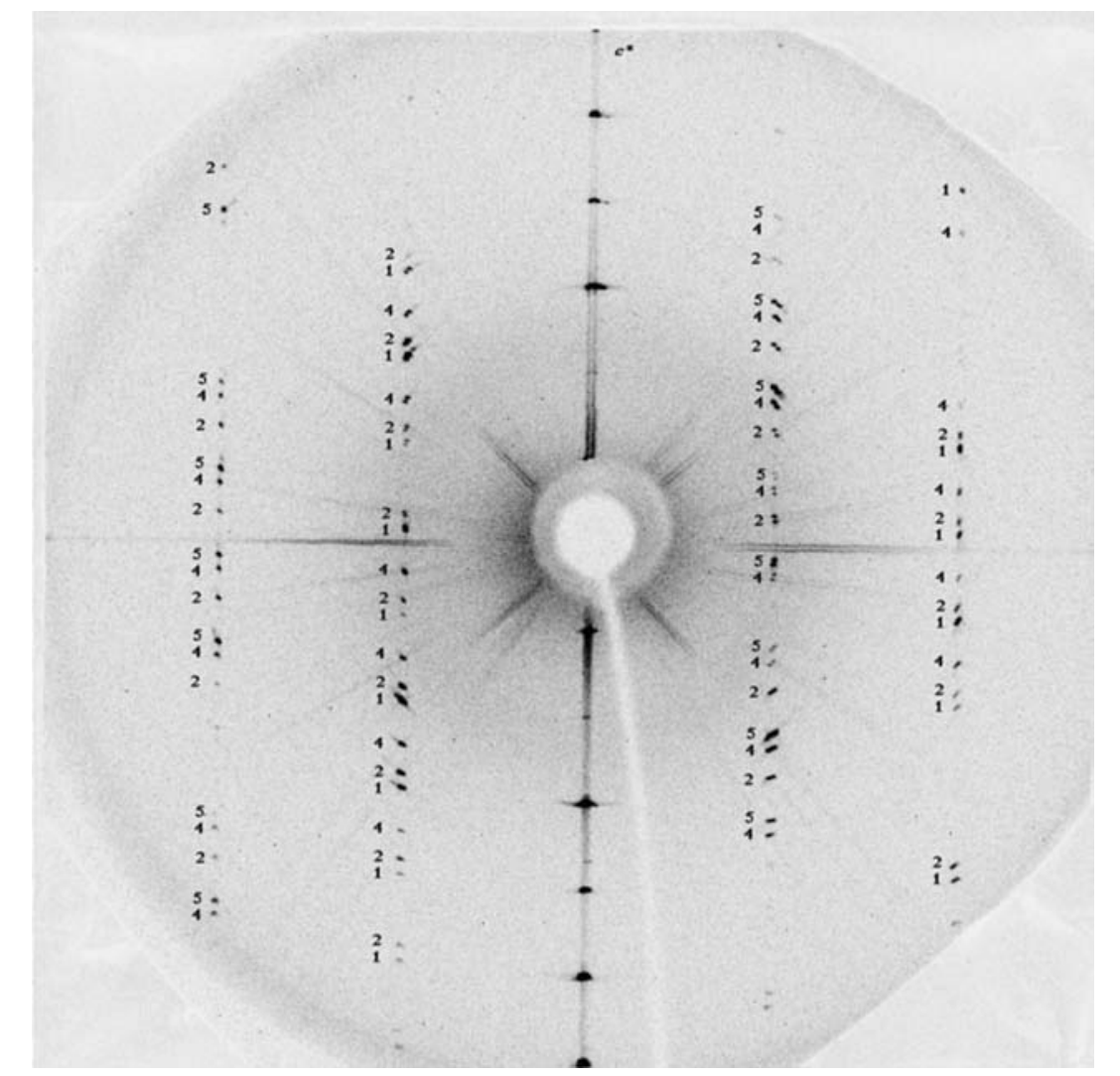

\section{Figure 9}

Precession diffraction pattern from the third SX plane (SX3) of the sample No. $2\left(120^{\circ}\right.$ from SX1). The $l$ index is as given in Fig. 2.

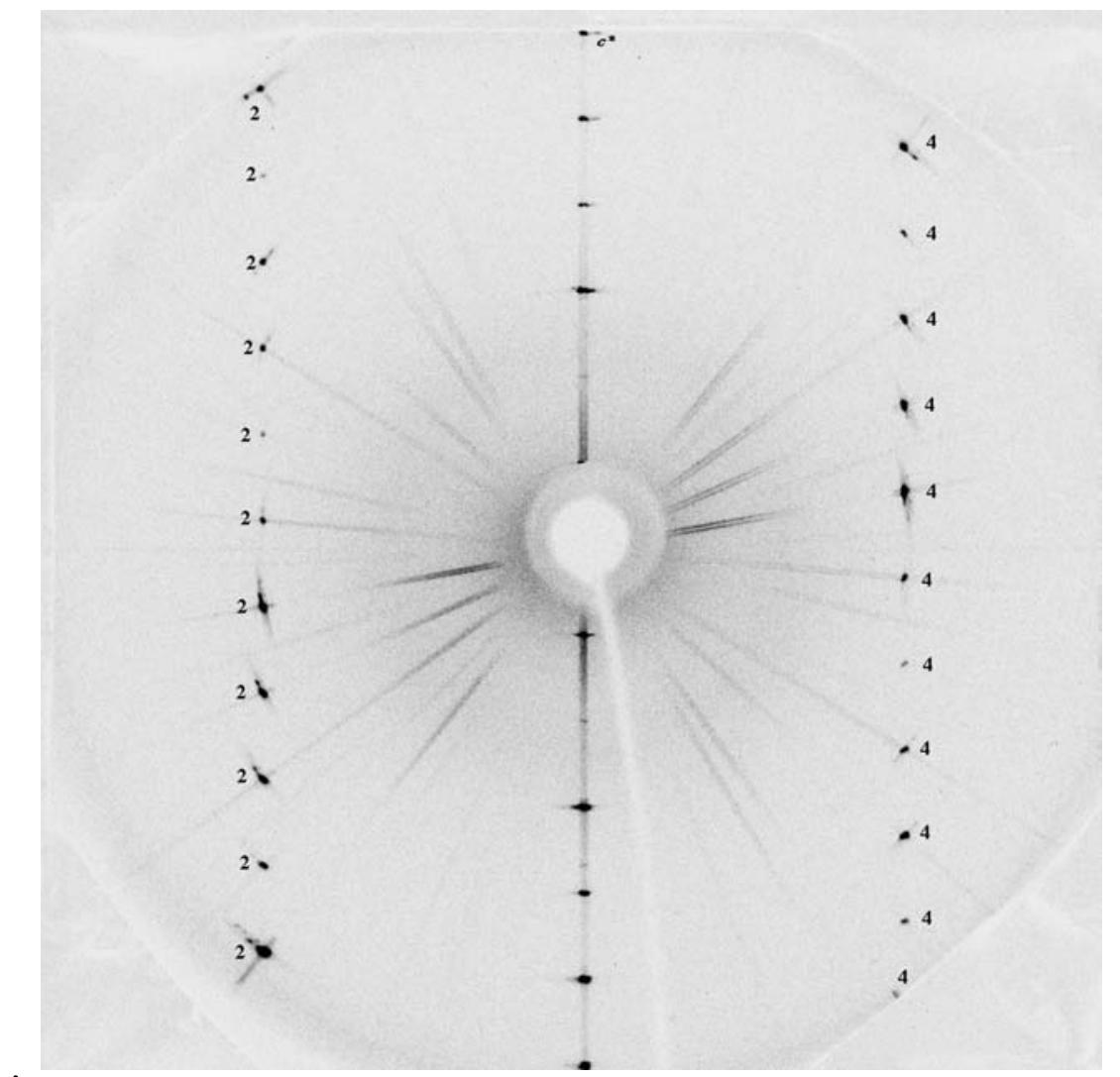

Figure 10

Precession diffraction pattern from an SD plane of the sample No. 2 ( $30^{\circ}$ from SX3). The $l$ index is as given in Fig. 2. is shown in Fig. 11. Proceeding exactly as for sample 1, the comparison of the experimental minimal rhombus in the six orientations with the computed minimal rhombi shows that the sample is not simply a twin of $2 M_{1}$, nor does it contains a $3 T$ polytype. It is thus a $1 M-2 M_{1}$ allotwin, whose minimal rhombus matches that computed for a binary $1 M-2 M_{1}$ allotwin with $60^{\circ}$ rotation between the two individuals and $Z_{T}={ }^{3}{ }_{4}$. As in sample 1 , the allotwin indices are 6 and 3 for $1 M$ and $2 M_{1}$, respectively.

For the same reasons as those explained in the previous paragraph, the allotwin $Z_{T}={ }_{4}^{3}$ could be equivalently described as $Z_{T}={ }_{2}^{3}$ and it can correspond to four allotwin laws: [110], [110] (rotation allotwins), (130) and (13̄0) (reflection allotwins).

\section{Discussion}

So far, allotwins have been previously reported in $\mathrm{Li}-\mathrm{Fe}$ micas from the Krušné hory mountains, Czech Republic (Rieder, 1970), and were termed 'epitactic overgrowth' of two polytypes of the same substance. This is instead the first report of allotwins in the Ruiz Peak oxybiotite, in which a wealth of polytypes and twins has already been found. Their identification has been possible only through the geometrical approach recently developed by Nespolo et al. (2000). Owing to the frequent occurrence of $1 M$ and $2 M_{1}$ polytypes in this locality, there are higher chances of finding allotwins of $1 M$ and $2 M_{1}$. It is thus possible that several other examples from the same locality might have been previously missed simply because their diffraction pattern could not be interpreted.

The geometrical analysis of the diffraction pattern exploited in this research gives the relative orientation between allotwinned individuals, but not an univocal allotwin law: in fact, there are four possible allotwin laws corresponding to each of the two identified allotwins, as explained above. The identification of the true allotwin law would require a morphological analysis.

It should be noted that in the $Z_{T}={ }^{3}{ }_{4}$ allotwin the two individuals are rotated by $60^{\circ}$, which is an uncommon rotation both in mica twins and in mica polytypes. Odd multiples of $60^{\circ}$ rotations in mica twins 


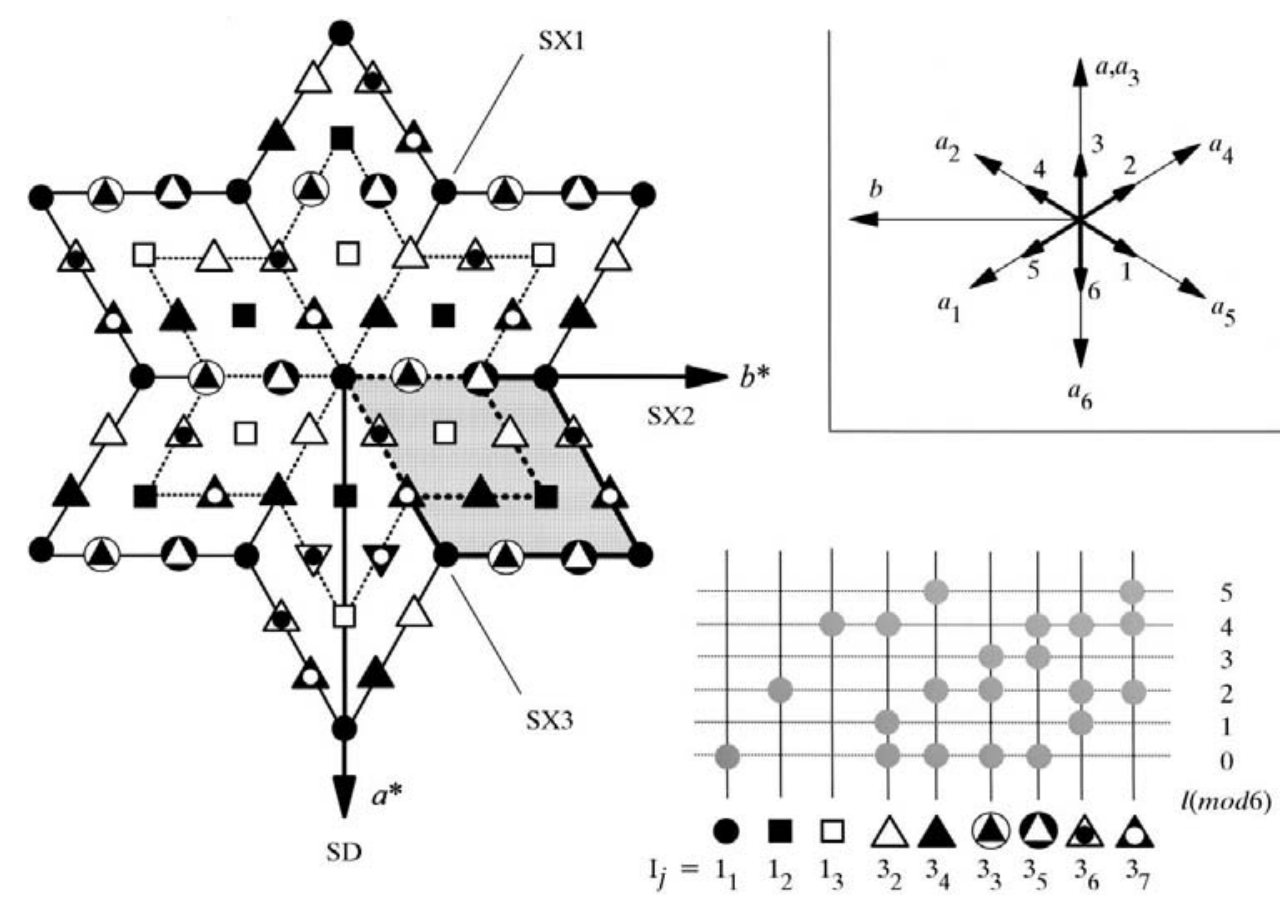

Figure 11

Construction of the star polygon corresponding to the diffraction patterns in Figs. 7-10 (see Fig. 6). The shaded minimal rhombus corresponds to the $Z_{T}={ }_{4}^{3}$ minimal rhombus tabulated in Nespolo et al. (2000).

have been reported by Sadanaga \& Takéuchi (1961), Bloss et al. (1963), Takeda \& Donnay (1965), Rieder (1970) and Sunagawa \& Tomura (1976), but are much rarer than the even multiples of $60^{\circ}$. For rotation twins the reason for the relative scarcity is likely to be the same, thus explaining the rarity of the odd rotations between layers in polytypes (Radoslovich, 1959): odd rotations produce a trigonal prismatic coordination of the interlayer cation on the interface between two individuals. In this type of coordination the $\mathrm{O}$ atoms are eclipsed, whereas for even rotations the coordination is trigonal antiprismatic, with a staggered configuration of the $\mathrm{O}$ atoms.

For reflection twins, since the composition plane is (quasi) normal to (001), odd rotations produce an interface with a low degree of coherency, as in the case of imperfect dislocations in the $2 M_{2}$ polytype (Baronnet \& Amouric, 1986). With the exception of the synthetic fluor-phlogopite reported by Bloss et al. (1963), all the quoted examples of odd rotations in twins, including the $Z_{T}={ }_{2}{ }_{2}$ allotwin presented here, have been found in natural micas formed in a fluid phase, where crystals are continuously moving. In this type of environment, the interaction and agglutination of crystals that had already attained a relatively large size is a common phenomenon (Sunagawa, 1977) and their relative adjustment is likely to be hindered even if they came into contact according to an energetically less stable odd configuration. Instead, the lower stability of the odd rotations is more easily avoided by slight movements when twins are formed during the nucleation stage, by smaller crystals. When agglutination of crystals that have already overcome the nucleation stage occurs, their relative adjustment is likely to be hindered and they tend to keep an orientation close to that by which they came into contact, even if it is energetically less stable. Sunagawa \& Tomura (1976) have experimentally demonstrated that agglutination of pre-formed crystals produces not only twins with both even and odd rotations, but also plesiotwins, which, we recall, are oriented crystal associations based on a large coincidence-site lattice and in which individuals are oriented with respect to each other by non-crystallographic rotations (Nespolo, Ferraris, Takeda \& Takéuchi, 1999). The interface energy increases from even rotations to odd rotations and to plesiotwin rotations.

This research has been partly developed at the Mineralogical Institute, University of Tokyo, during a Doctoral Program (MN, 1995-1998) supported by the Japanese Ministry of Education, Science and Culture. Part of this work (HT) has been undertaken at the Research Institute, Chiba Institute of Technology. MN acknowledges Professor Ichiro Sunagawa (Yamanashi Institute of Gemmology and Jewellery Arts) for profitable discussions; Professor Masamichi Miyamoto (University of Tokyo) and Professor Yasunari Watanabe (Teikyo Heisei University) for their constant encouragement. GF acknowledges financial support from the Italian National Research Council (CNR) and from the Italian Ministry of University and of Scientific and Technological Research (MURST, 40\% 'Micas' programme). The sample has been kindly provided by Dr Malcom Ross (US Geological Survey). The critical comments by two anonymous reviewers are acknowledged.

\section{References}

Bailey, R. A. \& Smith, R. L. (1978). New Mexico Bureau of Mines, Socorro, Circular 163, pp. 184-196.

Baronnet, A. \& Amouric, M. (1986). Bull. Mineral. 109, 489-508.

Bell, I. A., Wilson, C. J. L., McLaren, A. C. \& Etheridge, M. A. (1986). Tectonophysics, 127, 49-65.

Bloss, F. D., Gibbs, G. V. \& Cummings, D. (1963). J. Geol. 71, 537-547.

Dornberger-Schiff, K. \& Fichtner, K. (1972). Kristallogr. Technol. 7, 1035-1056.

Ďurovič, S., Weiss, Z. \& Backhaus, K.-O. (1984). Clays Clay Miner. 32, 454-474.

Fichtner, K. (1980). Match Commun. Math. Comput. Chem. 9, 21-40. Hahn, Th., Janovec, V. \& Klapper, H. (1999). Ferroelectrics, 222, 1121.

Hendricks, S. B. \& Jefferson, M. E. (1939). Am. Mineral. 24, 729-771. Hörz, F. (1970). J. Geophys. Res. 75, 965-977.

Ito, T. (1950). X-ray Studies on Polymorphism, 231 pp. Tokyo: Maruzen Co.

Kogure, T. \& Nespolo, M. (1999). Acta Cryst. B55, 507-516.

Nespolo, M. (1999). Mineral. J. 21, 53-85. 


\section{research papers}

Nespolo, M., Ferraris, G. \& Takeda, H. (2000). Acta Cryst. A56, 132148.

Nespolo, M., Ferraris, G., Takeda, H. \& Takéuchi, Y. (1999). Z. Kristallogr. 214, 378-382.

Nespolo, M., Kogure, T. \& Ferraris, G. (1999). Z. Kristallogr. 214, 5-8. Nespolo, M. \& Takeda, H. (1999). Mineral. J. 21, 103-118.

Nespolo, M., Takeda, H. \& Ferraris, G. (1998). Acta Cryst. A54, 348356.

Nespolo, M., Takeda, H., Ferraris, G. \& Kogure, T. (1997). Mineral. J. 19, 173-186.

Ohta, T., Takeda, H. \& Takéuchi, Y. (1982). Am. Mineral. 67, 298-310.

Radoslovich, E. W. (1959). Nature, 163, 253.

Rieder, M. (1970). Z. Kristallogr. 132, 161-184.

Rieder, M., Cavazzini, G., D'yakonov, Yu. S., Frank-Kamenetskii, V. A., Gottardi, G., Guggenheim, S., Koval', P. V., Müller, G., Neiva, A. M. R., Radoslowich, E. W., Robert, J. L., Sassi, F. P., Takeda, H., Weiss, Z. \& Wones, D. R. (1998). Can. Mineral. 36, 905-912.

Robbins, D. W. \& Strens, R. G. J. (1972). Mineral. Mag. 38, 551-563.
Ross, M., Takeda, H. \& Wones, D. R. (1966). Science, 151, 191-193. Ross, M. \& Wones, D. R. (1965). Am. Mineral. 50, 291.

Royer, L. (1928). Bull. Soc. Franç. Minér. Crist. 51, 7-159.

Royer, L. (1954). Bull. Soc. Franç. Minér. Crist. 77, 1004-1028. Sadanaga, R. \& Ohsumi, K. (1979). Acta Cryst. A35, 115-122.

Sadanaga, R. \& Takéuchi, Y. (1961). Z. Kristallogr. 116, 406-429.

Smith, J. V. \& Yoder, H. S. (1956). Mineral. Mag. 31, 209-235.

Smith, R. L., Bailey, R. A. \& Ross, C. S. (1961). US Geological Survey Professional Paper 366, 81 pp.

Sunagawa, I. (1977). J. Crystal Growth, 42, 214-223.

Sunagawa, I. \& Tomura, S. (1976). Am. Mineral. 61, 939-943.

Takano, Y. \& Takano, K. (1958). J. Mineral. Soc. Jpn, 3, 674-692 (in Japanese).

Takeda, H. \& Donnay, J. D. H. (1965). Am. Crystallogr. Assoc. Winter Meeting, Program and Abstracts, pp. 23-24.

Takeda, H. \& Ross, M. (1975). Am. Mineral. 60, 1030-1040.

Takeda, H. \& Ross, M. (1995). Am. Mineral. 80, 715-724.

Ungemach, H. (1935). Z. Kristallogr. 91, 1-22. 